\title{
Metabolic NMR mapping with microgram tissue biopsy
}

Covadonga Lucas-Torres ${ }^{1}$, Hélène Roumes ${ }^{2}$, Véronique Bouchaud ${ }^{2}$, Anne-Karine BouzierSore $^{2}$, Alan Wong ${ }^{1, *}$

1. NIMBE, CEA, CNRS, Université Paris-Saclay, CEA Saclay, 91191 Gif-sur-Yvette, France;

2. Centre de Résonance Magnétique des Systèmes Biologiques, CNRS-Université de Bordeaux, UMR5536 Bordeaux;

*corresponding author: (AW) alan.wong@ cea.fr, +33 (0) 148254054

Word count: 4,265 (13-pages) 
Abstract Summary: This study explores the potential of profiling $\mu$-scaled soft tissue biopsy by NMR spectroscopy. The important elements of high resolution and high sensitivity for the spectral data are achieved through a unique probe, HR- $\mu \mathrm{MAS}$, which allowed a comprehensive profiling to be performed on $\mu \mathrm{g}$-tissue for the first time under MAS conditions. Thorough spatially resolved metabolic maps were acquired across a coronal brain slice of rat C6 gliomas, which rendered the delineation of the tumor lesion. The results present a unique ex vivo NMR possibility to analyze tissue pathology that cannot be fully explored by the conventional approach, HR-MAS and in vivo MRS. Aside from the capability of analyzing a small localized region for tracking its specific metabolism, it could also offer the possibility to carry out longitudinal investigations on live animals due to the feasibility of minimally invasive tissue excision.

Keywords: metabolic mapping, HR- $\mathrm{MAS}$, NMR, glioma, biopsy.

\section{Abbreviations}

$\begin{array}{ll}A_{\text {met }} & \text { NMR peak Integral of a metabolite } \\ \text { AUC } & \text { Area Under the Curve } \\ \text { B } & \text { PLS coefficient matrix } \\ \text { C(n) } & \text { Control Slice } \\ \text { CPMGPR1D } & \text { Carr-Purcell-Meiboom-Gill with Presaturation } \\ \text { CVA } & \text { Canonical Variates Analysis } \\ \text { FWHM } & \text { Full-Width at Half-Maximum } \\ \text { HR-( } \mu \text { MAS } & \text { High-Resonance (micro) Magic-Angle Spinning } \\ \text { LASER } & \text { Localized by Adiabatic Selective Refocusing } \\ \text { Nmet }_{\text {m }} & \text { number of proton of a metabolite } \\ \text { PFA } & \text { Paraformaldehyde } \\ \text { PLS } & \text { Partial Least Square } \\ \text { OPLS-DA } & \text { Orthogonal Partial-Least Square Discriminant Analysis } \\ \text { ROC } & \text { Receiver Operating Characteristic } \\ \text { SNR } & \text { Signal-to-Noise Ratio } \\ \text { T(n) } & \text { Tumoral Slice } \\ \text { tCr } & \text { total Creatines } \\ \text { tCho } & \text { total Cholines } \\ \text { TurboRARE } & \text { Turbo-Rapid Acquisition with Refocused Echoes } \\ X_{\text {input }} & \text { PLS input matrix } \\ Y_{\text {output }} & \text { PLS output matrix }\end{array}$

\section{Introduction}


MRI has been a vital imaging modality for tumor diagnosis and prognosis due to its amenity of revealing the spatial contrast of the tissue in a non-invasive manner. However, it often relies on molecular spectroscopic technique, in vivo MRS, to unravel the underlying metabolic profiles and pathways for facilitating the characterization of the tumor subtypes and grades., ${ }^{1,2}$ Multivoxel MRS (MRS imaging) is a useful strategy in tissue pathology for providing the spatial metabolic coverage ${ }^{3,4}$ to understand cancer metabolic reprogramming. However, the spectral interpretation is generally strained by the insufficient resolution due to the acquisition under a static sample condition. Consequently, it commonly resorts to ex vivo HR-MAS NMR to support the interpretation of the in vivo spectra..$^{5-8}$

Despite the good spectral resolution by HR-MAS, there have been only few attempts of spatially resolved studies (multi-voxel or -sampling) in cancer research. Notably, Leo et al. performed the first HR-MAS analysis of different regions in a human glioblastoma. ${ }^{9}$ Recently, this team carried out a comprehensive metabolic mapping on a resected human prostate tumor. ${ }^{10}$ In another study, Righi et al. acquired a linear spatial metabolic profile across an excised rat glioma-bearing slice. ${ }^{11}$ However, one limitation with HR-MAS is the use of an extensive sampling mass (5-10 mg) for compensating the low sensitivity in NMR spectroscopy, which can hinder the deduction of relevant information in a small spatial region. Recently, a new $\mu \mathrm{MAS}$ probe technology, denoted as HR- $\mu \mathrm{MAS}$, has been introduced. ${ }^{12,13}$ It targets solely on $\mu \mathrm{g}$-scaled soft tissues with the capability of acquiring high resolution spectral data without compromising the sensitivity. Unlike the ultra-fast spinning $\mu \mathrm{MAS}$ probe for solids, the HR$\mu \mathrm{MAS}$ probe is devised to give minimal effect of the magnetic susceptibility toward the $\mu \mathrm{g}-$ tissue, allowing high-resolution acquisition to be achieved under the sample-spinning condition. This study examined the HR- $\mu$ MAS application to microbiopsies. It detailed the metabolic mapping of rat C6 glioma-bearing slices to facilitate the delineation of the tumor lesion. The C6 glioma model was strategically chosen for the proof of concept because of its homogenous nature and the well-characterized MR profiles, which allowed for an unbiased evaluation of the profiling capability of HR- $\mu \mathrm{MAS}$, which uses $\mu$-tissue.

\section{Experimental section}

\section{Animal treatment and glioma implantation}

Six male Wistar rats (160-220 g body weight) were used: two controls (C1 and C2), and four tumor-bearing rats (T1-T4). T1-T4 tumors were produced by stereotaxic injection in the left caudate nucleus (part of the basal ganglia) of C6 glioma cells, derived from $\mathrm{N}$ - 
nitrosomethylurea-induced rat glioblastoma. ${ }^{14}$ Rats were anesthetized with isofluorane (4\% induction, 2\% during surgery) and secured in the stereotaxic apparatus (Stoeling, Germany). The scalp was cleaned with Betadine (Sarget, France) and local injection of lidocaine was performed (local anesthesia). The skull was then exposed by a midline scalp incision and a 1$\mathrm{mm}$ diameter hole was drilled at a position 3.5-mm left from Bregma. Then, $10^{6}$ glioma cells in $10 \mu \mathrm{l}$ were injected into the left hemisphere at a depth of $5.5 \mathrm{~mm}$. The craniectomy was sealed with bone wax, and the scalp was sutured. In vivo MRI and MRS were periodically performed to follow the tumor growth.

\section{In vivo ${ }^{1} \mathrm{H}$ MRI and MRS}

MRI and MRS were performed on a 7 T Bruker BioSpec scanner (70/20, Ettlingen, Germany) using a Bruker ${ }^{1} \mathrm{H}$-transmit volume coil. The tumor was visualized using a $\mathrm{T}_{2}$-TurboRARE sequence (TE 39.96 ms; TR 2500 ms; average 2; repetition 1; echo spacing $13.319 \mathrm{~ms}$; rare factor 8 ; slice thickness $0.8 \mathrm{~mm}$; 20 slices; interspace $0.2 \mathrm{~mm}$; image size $256 \times 256$; field-ofview $35 \times 35 \mathrm{~mm}$; scan time $2 \mathrm{~min} 40 \mathrm{~s}$ ). ${ }^{1} \mathrm{H}$ MRS was performed using a LASER sequence (voxel $2.5 \times 2 \times 2.5 \mathrm{~mm}$; TE $19.26 \mathrm{~ms}$; TR $2.5 \mathrm{~s}$; average 256; acquisition time $12 \mathrm{~min}$ ). Acquisitions were performed with ParaVision 6.0. MRS spectral data were processed separately using Bruker Topspin 3.6.1.

\section{Slice resection}

Rats were euthanized using brain-focused microwaves ( $5 \mathrm{~kW}, 1 \mathrm{~s}$, Sacron8000, Sairem), which stopped all enzymatic and metabolic activities, to avoid post-mortem metabolism and artifacts such as anaerobic lactate production. ${ }^{15}$ Brains were then surgically removed, and a 3-mm coronal slice centered on Bregma was rapidly excised, placed in a biopsy cassette, and dipped in liquid nitrogen. The 3-mm thickness was chosen to accommodate a tissue-filled $\mu$ MAS rotor for an optimal NMR sensitivity. For T3 and T4, an additional slice (adjacent to that used in HR$\mu$ MAS) was excised for histopathology. Table S1 lists a summary of the slices (C1, C2 and T1T4) and their usages.

\section{Histopathology}

Histopathology was carried on the adjacent slice of T3 and T4 using a classical HematoxylinEosin staining protocol. Slices were immediately post-fixed for $24 \mathrm{~h}\left(4{ }^{\circ} \mathrm{C}\right.$, in PFA $\left.4 \%\right)$. Fixed slices were then cryoprotected by sucrose immersion $(48 \mathrm{~h}$ in a $20 \%$ sucrose solution, followed 
by $24 \mathrm{~h}$ in a $30 \%$ solution, $4{ }^{\circ} \mathrm{C}$ ) and frozen in liquid $\mathrm{N}_{2}$ vapor. The $20 \mu \mathrm{m}$-thick slices were stained with a hematoxylin solution (5 min), washed in distilled water (10 s), dipped in eosin solution (3 $\mathrm{min}$ ) and rinsed twice (in distilled water). Sections were finally dehydrated in $95 \%$ $(1 \mathrm{~min})$, then in $100 \%$ ethanol (2 min) and mounted on coverslips in Depex mounting medium (Sigma Aldrich). Brain sections were observed and images were taken using an optical microscope (Leica STP6000).

\section{ex vivo ${ }^{1} \mathrm{H}$ HR- $\mu$ MAS NMR}

Sample-preparation. Following the guidelines, ${ }^{16}$ the resected slice was placed inside a biopsy cassette with $1 \times 1 \mathrm{~mm}$ slots for imprinting the orderly grid pattern to facilitate the sampling (Figure S1). The sampling was carried out using a microbiopsy punch (World Precision Instruments) on a frozen slice, and immediately injected into the 1-mm rotor with few drops of $\mathrm{D}_{2} \mathrm{O} / \mathrm{TSP}$ (internal reference). The entire procedure was carried out on a cold platform (Figure S1) and limited to 15-20 minutes. Samples with a longer preparation were automatically discarded.

NMR acquisition. A sacrificed sample was used for optimization (shimming and pulsesequence adjustments). NMR experiments were carried out on a Bruker Avance II, operating at a ${ }^{1} \mathrm{H}$ frequency of $499.16 \mathrm{MHz}$ with a custom-built JEOL 1-mm 'HR'- $\mu$ MAS under a sample spinning between $5000-5500 \pm 20 \mathrm{~Hz}$, and with a constant temperature of $20 \pm 2{ }^{\circ} \mathrm{C}$. The NMR spectra were acquired with a Carr-Purcell-Meiboom-Gill (cpmgpr1d) pulse sequence (TE 80 ms; water-suppression recycle delay $1 \mathrm{~s}$; spectral width $20 \mathrm{ppm}$; data point $32 \mathrm{~K}$ ). Four subsequent spectra were collected for each sample, each with 320 averaging with a 13-minute acquisition period. The spectral processing was performed using Bruker Topspin 3.6.1 with a zero-filling and a line broadening of $0.3 \mathrm{~Hz}$. The ${ }^{1} \mathrm{H}$ chemical shift was referenced to the alanine doublet at $1.48 \mathrm{ppm}$. Figure $\mathrm{S} 2$ shows a comparison of the resolutions between the conventional HR-MAS approach and HR- $\mu$ MAS. It also shows no apparent signal degradation among the four subsequent spectra. The summed spectra were used in the multivariate and semiquantification analyses.

\section{Data analysis}

Multivariate. Processed spectral data were uploaded into MestreNova v8.1. Spectra were aligned, and the water region (4.15-5.10 ppm) was removed. Each spectrum was normalized to the signal of $\mathrm{tCr}$ (3.06 to $3.02 \mathrm{ppm}$ ), and divided into sub-ppm (0.01 ppm) buckets from 5.20 
to $0.77 \mathrm{ppm}$. The resultant matrix was submitted to SIMCA P13 and scaled by Pareto's distribution (power-law probability distribution). PCA and OPLS-DA were performed to identify the tumor profile. PLS regression was applied to locate the lesion. Models were validated by assessing the values of $R^{2} X, R^{2} Y$, and $Q^{2}$ as well as by the $p$-values obtained from cross-validation (level of confidence $<0.05$ ) to avoid overfitting. It should be noted that we applied PLS regression because of the accessibility in SIMCA (other regressions algorithms can offer similar, or better, prediction accuracy, such as a combined PCA and canonical variates analysis (CVA), which has been used on the MRS imaging data for delineating prostate tumors in an excised human tissue ${ }^{3}$.

Semi-Quantification. Due to the differences in the spin relaxation $\left(\mathrm{T}_{1}\right.$ and $\left.\mathrm{T}_{2}\right)$ of metabolic signals, we reported the metabolic ratio $Q[$ met $A /$ metB $]=\left(A_{\text {met }} / A_{\text {metB }}\right) \times\left(N_{\text {metB }} / N_{\text {metA }}\right)$, where $A_{\text {met }}$ and $\mathrm{N}_{\text {met }}$ are the corresponding signal integral and its number of detected protons, respectively. The deconvolution was carried out by MestreNova v8.1. A representative fitting is shown in Figure S3, and the quantified ratios are summarized in Tables S2-4.

\section{Results and discussion}

\section{HR- $\mu$ MAS NMR of brain tissue}

Figure 1 shows a spectral comparison between ex vivo HR- $\mu$ MAS NMR and in vivo MRS with a sampling voxel of $1 \times 1 \times 3 \mathrm{~mm}$ for HR- $\mu$ MAS and $2.5 \times 2 \times 2.5 \mathrm{~mm}$ for MRS. Despite the fact that acquisitions were carried out at different magnetic fields ( $7 \mathrm{~T}$ for in vivo MRS and $11.7 \mathrm{~T}$ for HR- $\mu \mathrm{MAS}$ ), the spectral effect of MAS is evident. The FWHM of a singlet at $3 \mathrm{ppm}$ in HR$\mu \mathrm{MAS}$ is significantly narrower with $0.02 \mathrm{ppm}$ as compared to that of $0.6 \mathrm{ppm}$ in in vivo MRS. Moreover, with the same acquisition time, the SNR for HR- $\mu \mathrm{MAS}$ is higher than that for in vivo MRS. Taking into account the sampling voxel, HR- $\mathrm{MAS}$ renders a crude 7-fold enhancement in volume-sensitivity $\operatorname{SNR}\left(\mathrm{mm}^{3} \cdot \sqrt{ } \mathrm{min} \cdot \sqrt{ } \mathrm{B}^{3}\right)$. In other words, it would require a $7^{2}$, therefore a 49, longer acquisition time for in vivo MRS to achieve the same SNR level obtained with HR- $\mu$ MAS with $\mu$ g-tissue volume. This enhancement is ascribed by the use of a $\mu$-detection solenoid and the resultant spectral resolution achieved by the HR- $\mu$ MAS probe. Note that the $\mu$ MAS for solids would not offer the same level of resolution because of the presence of the substantial magnetic susceptibility that will arise from the probe head.

\section{Metabolic NMR profile of gliomas}


PCA and OPLS-DA were carried out to identify a specific glioma metabolic profile by examining relative metabolite intensities between the tumor and control tissues. Owing to the $\mu \mathrm{g}$-scaled sampling, this study was able to analyze a total of 34 samples from a single control coronal slice (C1) and 42 from two glioma-bearing slices (T1 and T2) (Figure S1). The PCA score plot in Figure 2 exhibits a clear distinction of spectral data between control and tumor slices. The discrimination was also reflected in the OPLS-DA with $\mathrm{R}^{2} \mathrm{X}=0.843$ and $\mathrm{Q}^{2}=0.752$, and with a modest predictive accuracy $\mathrm{AUC}=85 \%$. Owing to the homogenous nature of the tumor tissue and its distinct metabolic profile, the discrimination was further improved between the data acquired from the tumor lesion alone and the control with $\mathrm{R}^{2} \mathrm{X}=0.986, \mathrm{Q}^{2}=0.963$ and with a near $100 \%$ AUC; thus, the corresponding S-line has unambiguously diagnosed the NMR markers for the tumor: elevated signals in tCho, Tau, Ala, Gly, myo-Inositol, Lac and lipids, while a reduction in NAA and Glu signals were observed. These results are in line with the previous HR-MAS findings, ${ }^{17}$ thus validating the NMR profiling strategy with HR- $\mu$ MAS.

\section{Glioma delineation and characterization}

To evaluate the possibility of localizing the tumor lesion through the spatial NMR mapping, we used a linear PLS regression, $\boldsymbol{Y}_{\text {output }}=\boldsymbol{X}_{\text {input }} \boldsymbol{B},{ }^{18}$ by exploiting the $100 \%$ AUC model (Figure 2c) as the regression coefficient $\boldsymbol{B}$ to delineate the lesion in the T3 and T4 models. This was carried out by mapping the NMR profiles across the coronal slices, in which the acquired spectral data $\boldsymbol{X}_{\text {input }}$ were then submitted to the regression. The outputs $\boldsymbol{Y}_{\text {output }}$ provide the predictive degree 01, where 1 represents the spectral profile closely resembling those of the lesion used in $\boldsymbol{B}$ (T1 and T2), and likewise, 0 corresponds to those of the control (C1). Figure 3 shows the resulting regression maps. Evidently, the test model C2 is solely mapped with the control profiles, whereas the located tumor lesions are apparent in T3 and T4, which are in agreement with the visible tumor mass on MR images. Interestingly, the regression indicates that the lesion profiles delineated in T4 are more similar to those in $\boldsymbol{B}(\mathrm{T} 1, \mathrm{~T} 2)$ as compared to those in T3.

The study quantified and evaluated several NMR-identifiable metabolites across the coronal slice of C2, T3 and T4. Figure 4 shows that in both T3 and T4 an increase in tCho/NAA, $\mathrm{Lac} / \mathrm{NAA}$, Gly/tCr and $\mathrm{Ala} / \mathrm{tCr}$, and a decrease in $\mathrm{Glu} / \mathrm{tCr}$ were observed in the lesion. In general, the elevated tCho/NAA arises both from a decrease in NAA signal and an increase in tCho. This magnetic resonance signature has been well recognized and often used as a diagnostic marker for characterizing tumoral tissue in animal models ${ }^{6,11}$ and in humans..$^{1,5,6,18}$ The reduction in NAA, a neuronal viability marker, is interpreted as the loss or abnormality of 
the neuronal tissue, whereas the increased level of tCho is associated with membrane synthesis and degradation (such as occurring during necrosis, for example). Among tCho signal ratios, T4 displays a slightly higher PC/tCho level $(0.69 \pm 0.09$, Table 1$)$ at the lesion along with a lower level of GPC/tCho $(0.24 \pm 0.09)$ as compared to those in $\mathrm{T} 3(0.56 \pm 0.04$ and $0.38 \pm 0.06$, respectively). In a previous study on choline metabolism using ex vivo HR-MAS NMR, ${ }^{19}$ it has been found a difference of the PC level and GPC in human tissues between high and low grade astrocytomas, which appeared to be reflected by the changes in the expression of genes of the Kennedy pathway.

A recent HR-MAS study ${ }^{11}$ has found that the tumor lesion is metabolically dominated by anaerobic glycolysis (Warburg effect), which is reflected by the production of lactate from pyruvate through lactate dehydrogenase activity. The study has also observed a decrease in glutamate, presumably reflecting neuronal death, since glutamate is the major neurotransmitter in glutamatergic neurons. ${ }^{20}$ These results (an increase in lactate and a decrease in Glu) are also evident in Figure 4. An increasing level of $\mathrm{Glu} / \mathrm{tCr}$ from the peritumor to the contralateral region was apparent in both T3 and T4. Interestingly, the growth was more pronounced in T4, where this ratio in the contralateral hemisphere was even higher compared to the one in $\mathrm{C} 2$. This alteration has also been observed in the edema surrounding different human gliomas, ${ }^{21}$ and could signify neuronal death due to a toxic effect of the excess of excitatory neurotransmitter (exitotoxicity). The high content of Lac/NAA found in the T4 lesion suggests a higher grade glioma, which is often accompanied by lactate production that diffuses from the tumor to the peritumoral region. ${ }^{22}$

Glycine (Gly) is an inhibitory neurotransmitter. It has been found to increase in high grade gliomas by in vivo MRS ${ }^{23}$ and ex vivo HR-MAS NMR, ${ }^{24}$ and has been proposed as a tumoral marker. Despite the $\mu \mathrm{g}$-scaled tissue, the achieved high resolution and sensitivity of HR- $\mu \mathrm{MAS}$ enable to capture the elevated level of Gly/tCr in the lesions.

An elevated level of Ala/tCr in the lesion was also detected, especially in T4. This is consistent with the previous HR-MAS findings in human meningiomas. ${ }^{17}$ It was suggested in an early study $^{25}$ that Ala inhibits the pyruvate kinase in gliomas and meningiomas, and Ala has been proposed as a potential biomarker for the evaluation of tumor aggressiveness due to its positive presence in glioblastoma multiforme as compared with low grade gliomas. ${ }^{19}$

The tumor grade is determined by the degree of differentiation of the tumor and its cellularity, as well as the presence of cytonuclear atypia, mitotic activity, microvascular proliferation and necrosis. As such, molecular characterization of the gliomas is an important aspect in clinical 
medicine for making diagnostic and treatment decision based on the grade. In vivo MRS provides metabolic information of the intratumoral region and facilitates distinguishing the glioma grades. The typical markers are tCho, NAA, Cr and lactate, and the lesser common are Gly, myo-Inositol, PC and GPC. For examples, increase in ratios of tCho/NAA, PC/tCho, Gly/tCr and in lipids, and reduction of the GPC/tCho ratio are consistently found in high grade compared to low grade gliomas.

Here, we evaluated the relevant metabolic ratios of the identified lesion in T1-T4. Ratios are summarized in Table 1 and Figure 5 , and suggest the following grade ranking $\mathrm{T} 1 \approx \mathrm{T} 2 \approx \mathrm{T} 4>$ T3. The higher grade in T4 compared with T3 is also evident in the histopathology shown in Figure 5b, in which angiogenesis, necrosis and hemorrhages are observed in T4 but not in T3. Regrettably, histopathology for T1 and T2 were not available to confirm the comparative grades. Interestingly, despite the four glioma-bearing rats underwent the same surgical stereotactic C6-cell implantation, the tumor growth was evidently different; therefore leading to different tumor metabolic patterns among the rats, especially for T3. Similar observations have also been reported. ${ }^{26}$ The previous study - using the same tumor implantation protocol also observed two distinct patterns of C6 glioma growth; one with capillaries found at the tumor periphery, and the other containing a tumor core in the center, reflecting the different tumor metabolism and necrosis processes.

Table 1. A summary of the mean metabolite ratios deduced from the delineated tumor lesion. ${ }^{\mathrm{a}}$

\begin{tabular}{|c|c|c|c|c|c|c|c|c|c|}
\hline & Lac/NAA & tCho/NAA & GPC/tCho & $\mathrm{PC} / \mathrm{tCho}$ & $\mathrm{Glu} / \mathrm{tCr}$ & $\mathrm{GABA} / \mathrm{tCr}$ & Gly/tCr & $\mathrm{Tau} / \mathrm{tCr}$ & $\mathrm{Ala} / \mathrm{tCr}$ \\
\hline $\mathrm{T} 1(\mathrm{n}=7)$ & $5.59 \pm 2.09$ & $6.61 \pm 0.35$ & $0.24 \pm 0.14$ & $0.68 \pm 0.12$ & $0.67 \pm 0.08$ & $0.24 \pm 0.02$ & $0.36 \pm 0.01$ & $0.78 \pm 0.05$ & $0.40 \pm 0.01$ \\
\hline $\mathrm{T} 2(\mathrm{n}=7)$ & $1.87 \pm 1.01$ & $8.17 \pm 1.36$ & $0.29 \pm 0.08$ & $0.65 \pm 0.07$ & $0.82 \pm 0.31$ & $0.17 \pm 0.12$ & $0.37 \pm 0.09$ & $0.90 \pm 0.17$ & $0.39 \pm 0.11$ \\
\hline$T 4(n=6)$ & $4.16 \pm 1.49$ & $6.30 \pm 2.39$ & $0.24 \pm 0.09$ & $0.69 \pm 0.09$ & $1.19 \pm 0.13$ & $0.48 \pm 0.12$ & $0.32 \pm 0.13$ & $0.87 \pm 0.16$ & $0.39 \pm 0.07$ \\
\hline$T 3(n=4)$ & $1.78 \pm 0.24$ & $2.26 \pm 0.52$ & $0.38 \pm 0.06$ & $0.56 \pm 0.04$ & $0.70 \pm 0.14$ & $0.16 \pm 0.06$ & $0.27 \pm 0.03$ & $0.75 \pm 0.06$ & $0.30 \pm 0.0$ \\
\hline $\mathrm{C} 2(\mathrm{n}=11)^{\mathrm{b}}$ & $0.56 \pm 0.21$ & $0.99 \pm 0.25$ & $0.31 \pm 0.12$ & $0.54 \pm 0.10$ & $1.07 \pm 0.15$ & $0.31 \pm 0.2$ & $0.08 \pm 0.02$ & $0.44 \pm 0.04$ & $0.12 \pm 0.02$ \\
\hline $\mathrm{C} 1(\mathrm{n}=13)^{\mathrm{b}}$ & $0.75 \pm 0.63$ & $0.94 \pm 0.27$ & $0.28 \pm 0.11$ & $0.55 \pm 0.06$ & $0.91 \pm 0.20$ & $0.34 \pm 0.08$ & $0.10 \pm 0.03$ & $0.46 \pm 0.09$ & $0.13 \pm 0.05$ \\
\hline
\end{tabular}

(a) Test models are highlighted in grey; (b) Taken from the contralateral hemisphere.

\section{Concluding Remarks}

This work successfully demonstrates the metabolic profiling of $\mu \mathrm{g}$-scaled soft tissue biopsy under MAS conditions. As a proof of concept, the delineation of the tumor lesion in the gliomabearing rat was carried out by exploiting the new $\mu$ MAS technology, which permitted highresolution NMR data acquisition of small tissue voxel. These results illustrate a potential toward the acquisition of metabolic information on a small spatial origin; data that can be difficult to obtain from the conventional HR-MAS and in vivo MRS experiments alone. We foresee suitability for examining the spatial patterns of local drug release in the region of interest. Also, 
the notion of $\mu$ g-scaled tissue excision promotes minimally invasive surgical procedure on animals, which could present a unique possibility of acquiring longitudinal data on live animals. Like in all analytical technology, limitations are unavoidable. In HR- $\mu$ MAS, the low detection sensitivity is an intrinsic bottleneck. Despite the fact that $\mu$-solenoid provides an enhancement for $\mu$ g-tissue analysis, it is still insufficient to analyze low abundant metabolites. To further improve the sensitivity, it needs to be coupled with other enhancement strategy. One would be the cryogenic probe technology, which reduces the thermal noise originating from the electronic circuits. However, the change in the coil susceptibility under the cryo-temperature would greatly complicate the attainment of high resolution data. Another limitation is the sample preparation, since handling a $\mu$ g-scaled tissue with a tiny rotor is not trivial. A slight deviation could affect the overall data, resulting in non-reproducible data acquisition and consequently would lead to misinterpreted analysis. Therefore, a more robust and automated approach to fill the rotor would enhance its feasibility and reliability, and would facilitate transfer to biopsy research. In spite of these limitations, the remarkable ability of profiling $\mu \mathrm{g}$-scaled biopsies promotes a new possibility of assessing tissue pathology and could lead to a valuable complementary option to aid data interpretation of in vivo MRS and ex vivo HR-MAS NMR.

Supplementary Materials: Figures include sampling coordinates for HR- $\mu$ MAS, representative HR- $\mu$ MAS spectra and spectral fitting. Summary tables include the resected brain slices, and deduced metabolite ratios in T3, T4 and $\mathrm{C} 2$.

Author Contributions: CLT and AW designed and constructed the study, and carried out the NMR measurements and data analyses. AKBS, HR and VB carried out the animal treatments, MRI and data interpretations. CLT and AW drafted the manuscript, and all authors assisted in revisions.

Funding: The study was supported by Agence Nationale de la Recherche in France, grant number ANR-16-CE11-0023. AKBS also received support from ANR-10-IDEX and ANR-10LABX-57.

Acknowledgments: We are grateful to JEOL Resonance Inc. for their technical supports on the HR- $\mu$ MAS probe, and to Prof Luc Pellerin (Univeristé de Poitiers, France) and Dr Yusuke Nishiyama (JEOL, Japan) for reviewing the manuscript. We would also like to thank Dr Florence Fauvelle (GIN, France) for advising about sample preparation.

Conflicts of Interest: The authors declare no conflict of interest.

Data availability: The data that support the findings of this study are available from the 
corresponding author upon reasonable request.

\section{Reference}

1. Horská A, Barker PB. Imaging of brain tumors: MR spectroscopy and metabolic imaging. Neuroimag Clin N Am. 2010;20:293-310.

2. Mandal PK. Brain metabolic mapping with MRS: A potent noninvasive tool for clinical diagnosis of brain disorders. Am J Neuroradiol. 2014;35:S1

3. Wu C-L, Jordan K.W, Ratai EM, et al. Metabolomic imaging for human prostate cancer detection. Sci Transl Med. 2010;2:16ra8.

4. Taylor L, Fuss BA, Cheng LL. Metabolic Imaging in Humans. Topic in Magn Reson Imaging 2016;25:223-235.

5. Opstad KS, Wright AJ, Bell BA, et al. Correlations between in vivo ${ }^{1} \mathrm{H}$ MRS and ex vivo ${ }^{1} \mathrm{H}$ HRMAS metabolite measurements in adult human gliomas. J Magn Reson Imaging 2010;31:289-297.

6. (a) Kaebisch E, Fuss TL, Vandergrift LA, et al. Applications of high-resolution magic angle spinning MRS in biomedical studies I-cell line and animal models. NMR Biomed. 2017; e3700. (b) Dietz C, Ehret F, Palmas F, et al. Applications of high-resolution magic angle spinning MRS in biomedical studies II-human diseases. NMR Biomed. 2017;e3784.

7. Tugnoli V, Schenetti L, Mucci A, et al. A comparison between in vivo and ex vivo HRMAS 1H MR spectra of a pediatric posterior fossa lesion. Int J Mol Med. 2005;16:3017.

8. Tugnoli V, Schenetti L, Mucci A, et al. Ex vivo HR-MAS MRS of human meningiomas: a comparison with in vivo 1H MR spectra. Int J Mol Med. 2006;18:859-69.

9. Cheng LL, Anthony DC, Comite AR, et al. Quantification of microheterogeneity in glioblastoma multiforme with ex vivo high-resolution magic-angle spinning (HRMAS) proton magnetic resonance spectroscopy. Neuro-Onco. 2000;87-95.

10. Dinges SS, Vandergrift LA, Wu S, et al. Metabolomic prostate cancer fields in HRMASMRS-profiled histologically benign tissue vary with cancer status and distance from cancer. NMR Biomed. 2019; 4038 .

11. Righi V, García-Martín M-L, Mucci A, et al. Spatially resolved bioenergetics and genetic reprogramming through the brain of rats bearing implanted C6 gliomas as detected by multinuclear high-resolution magic angle spinning and genomic analysis. $J$ Proteome Res. 2018;17:2953-2962.

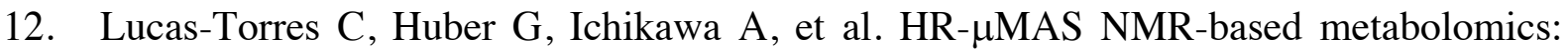
localized metabolic profiling of a garlic clove with $\mu \mathrm{g}$ tissues. Anal Chem. 2018;90:13736-13743.

13. Lucas-Torres C, Wong A. Current Developments in uMAS NMR analysis for metabolomics. Metabolites 2019;9:29.

14. Bendaz P, Lightbody J, Sato G, Sweet W. Differentiated rat glial cell strain in tissue culture. Science 1968;161:370-371. 
15. Sampol D, Ostrofet E, Jobin M-L, et al. Glucose and lactate metabolism in the awake and stimulated rat: a ${ }^{13} \mathrm{C}-\mathrm{NMR}$ study. Front Neuroenergetics 2013:5.

16. Lucas-Torres $\mathrm{C}$, Bernard $\mathrm{T}$, Huber $\mathrm{G}$, et al. General guidelines for sample preparation strategies in HR- $\mu$ MAS NMR-based metabolomics of microscopic specimens. Metabolites 2020;10:54.

17. AJ, Fellows GA, Griffiths JR, et al. Ex-vivo HRMAS of adult brain tumours: metabolite quantification and assignment of tumour biomarkers. Mol Cancer 2010;9:66.

18. Lee LC, Liong C-Y, Jemain AA. Patial least squares-discriminant analysis (PLS-DA) for classification of high-dimensional (HD) data: a review of contemporary practice strategies and knowledge gaps. Analyst 2018:143;3526-3539.

19. Righi V, Roda JM, Paz J, et al. ${ }^{1} \mathrm{H}$ HR-MAS and genomic analysis of human tumor biopsies discriminate between high and low grade astrocytomas. NMR Biomed. 2009;22:629-637.

20. Rothman DL, Behar KL, Hyder F, Shulman RG. In vivo NMR studies of the glutamate neurotransmitter flux and neuroenergetics: implications for brain function. Annu Rev Physiol. 2003:65;401-427.

21. Ricci R, Bacci A, Tugnoli V, et al. Metabolic findings on $3 \mathrm{~T}{ }^{1} \mathrm{H}-\mathrm{MR}$ spectroscopy in peritumor brain edema. Am J Neuroradiol. 2007:28;1287-1291.

22. Raschke F, Barrick TR, Jones TL, et al. Tissue-type mapping of gliomas. NeuroImage: Clin. 2019:21;101648

23. Maudsley AA, Gupta RK, Stoyanova R, et al. Mapping of glycine distributions in gliomas. Am J Neuroradiol. 2014:35;S31-S36.

24. Righi V, Andronesi OC, Mintzopoulos D, et al. High-resolution magic angle spinning magnetic resonance spectroscopy detects glycine as a biomarker in brain tumors. Int $J$ Oncol 2010;36:301-306.

25. Van Veelen CWM, Verbiest H, Staal GEJ, Vlug AMC. Alanine inhibition of pyruvate kinase in gliomas and meningiomas: A diagnostic tool in surgery for gliomas? Lancet 1977:384-385.

26. Fonchy E, Lahrech H, Francois-Joubert A, et al. A new gadolinium - based contrast agent for magnetic resonance imaging of brain tumors: Kinetic study on a C6 rat glioma model. J Magn Reson Imag. 2001;14:97-105. 


\section{Figure caption}

Figure 1. Spectral comparison between in vivo MRS (at $7 \mathrm{~T}$ ) and ex vivo HR- $\mathrm{MMAS}$ NMR (at $11.7 \mathrm{~T}$ ). Spectra correspond to the tumor lesion in $\mathrm{T} 2$. Both were acquired with an acquisition time of 13 minutes, but with different sampling voxel, $2.5 \times 2 \times 2.5 \mathrm{~mm}$ and $1 \times 1 \times 3 \mathrm{~mm}$, respectively.

Figure 2. (a) PCA and (b) OPLS-DA score plots between all sampling data acquired from C1 and T1-T2; (c) OPLS-DA plot between all data in C1 and those in the tumor lesion of $\mathrm{T} 1$ and T2; (d) ROC comparison between models in (b) and (c); and (e) OPLS-DA S-line plot of (c). The significant metabolites are annotated with a correlation coefficient $\mathrm{p}$ [corr] in parentheses and a Student's t-test $\mathrm{p}$-value $(* \mathrm{p}<0.01, * * \mathrm{p}<0.001$ and $* * * \mathrm{p}<0.0001)$

Figure 3. PLS-regression analyses: (a) A scheme of PLS regression, with the model in Figure 2c as $\boldsymbol{B}$; (b) in vivo MRI images of the corresponding T3 and T4; (c) Superimposed regression maps with $1 \times 1$ sampling pixels. The level of the predicted values is coded with a 32 -colour scheme with red being 1 , the likeliness to the tumor profile in $\boldsymbol{B}$, and blue being 0 , the likeliness to the control profile.

Figure 4. (a) Superimposed metabolite distribution maps across the coronal slice; (b) Linear plots showing the variations of the metabolite ratios across the coronal slice, along the sampling row 5-6 in Figure 3c. The colored regions correspond to the same scheme as in the regression maps Figure 3c.

Figure 5. (a) Bar graphs showing the comparison of metabolite ratios found within tumor regions. Values correspond to the arithmetic mean and the error bar to its standard deviation, and are summarized in Table 1. The t-test's p-values are indicated as $* \mathrm{p}<0.05$ and $* * * \mathrm{p}<$ 0.0001; (b) Hematoxylin-Eosin histological sections of the adjacent glioma-bearing slices. The corresponding $250 \mu \mathrm{m}$ scale-bar is displayed in the upper images $(\times 2.5)$ and in lower images (×10). 
in vivo MRI \& MRS @ 7T
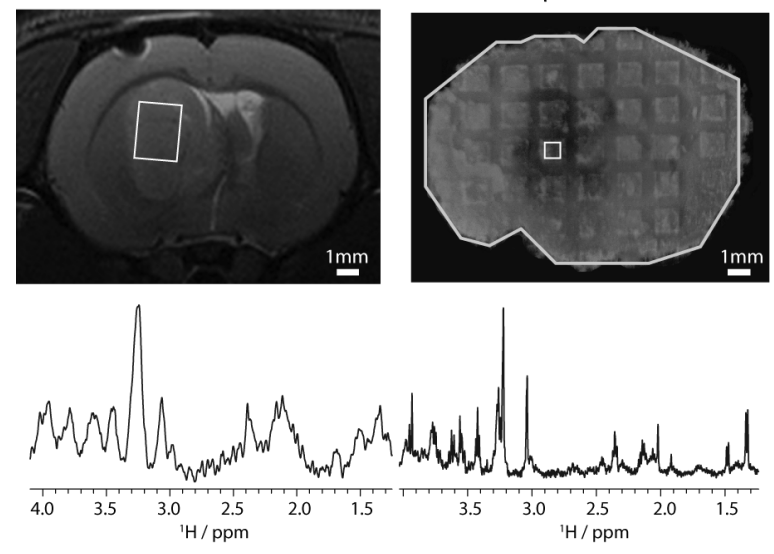

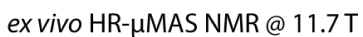

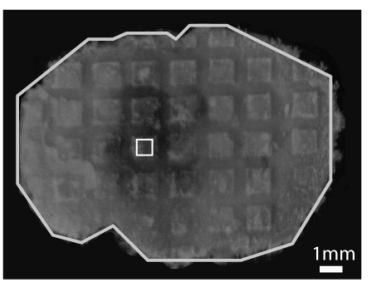

Figure 1 (a) Data in control slice C1 ( $\mathrm{n}=34)$

a) $\square \square$ Data in tumor slice T1,T2 ( $n=42)$

$\square \quad$ Tumor lesion data in T1,T2 (n=14)

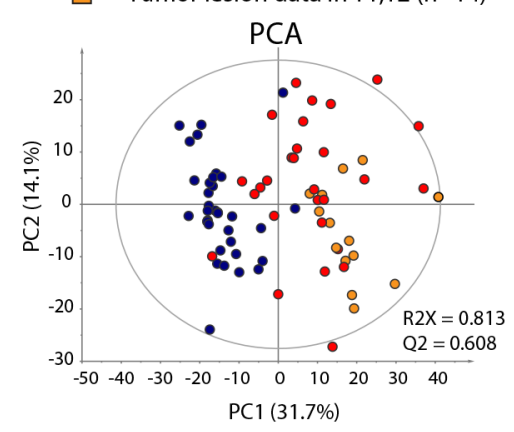

(d)

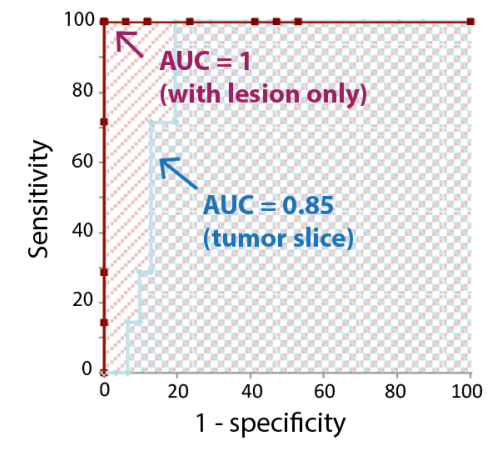

(b)

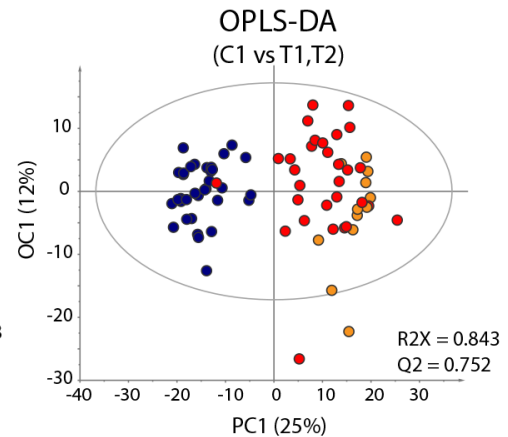

(e)

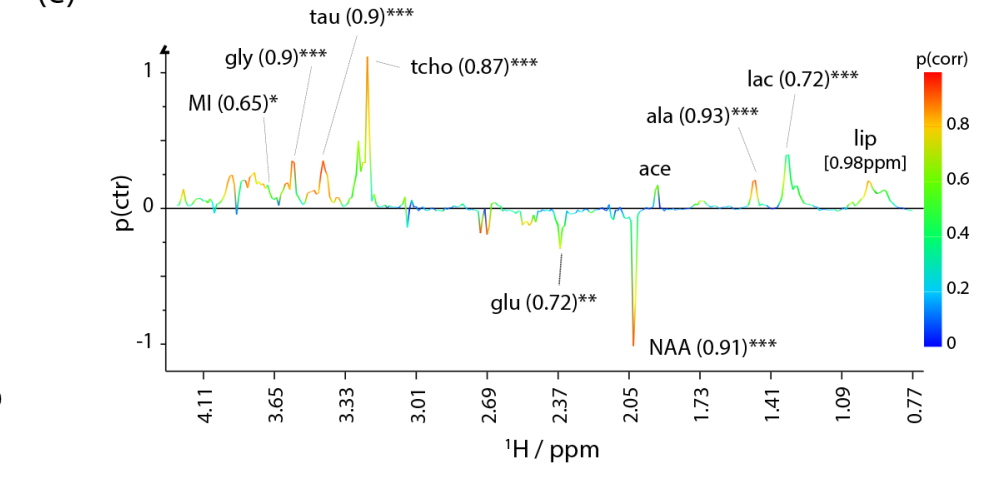

(c)

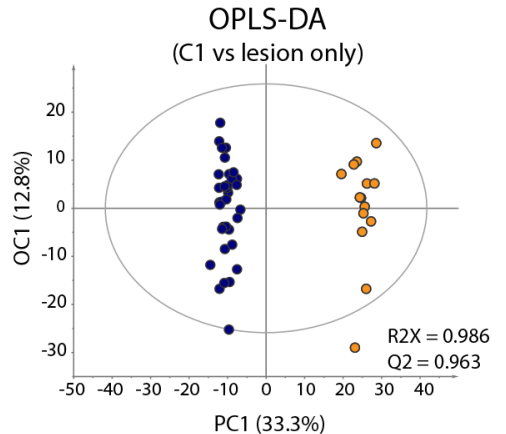

PC1 (33.3\%)

Figure 2 


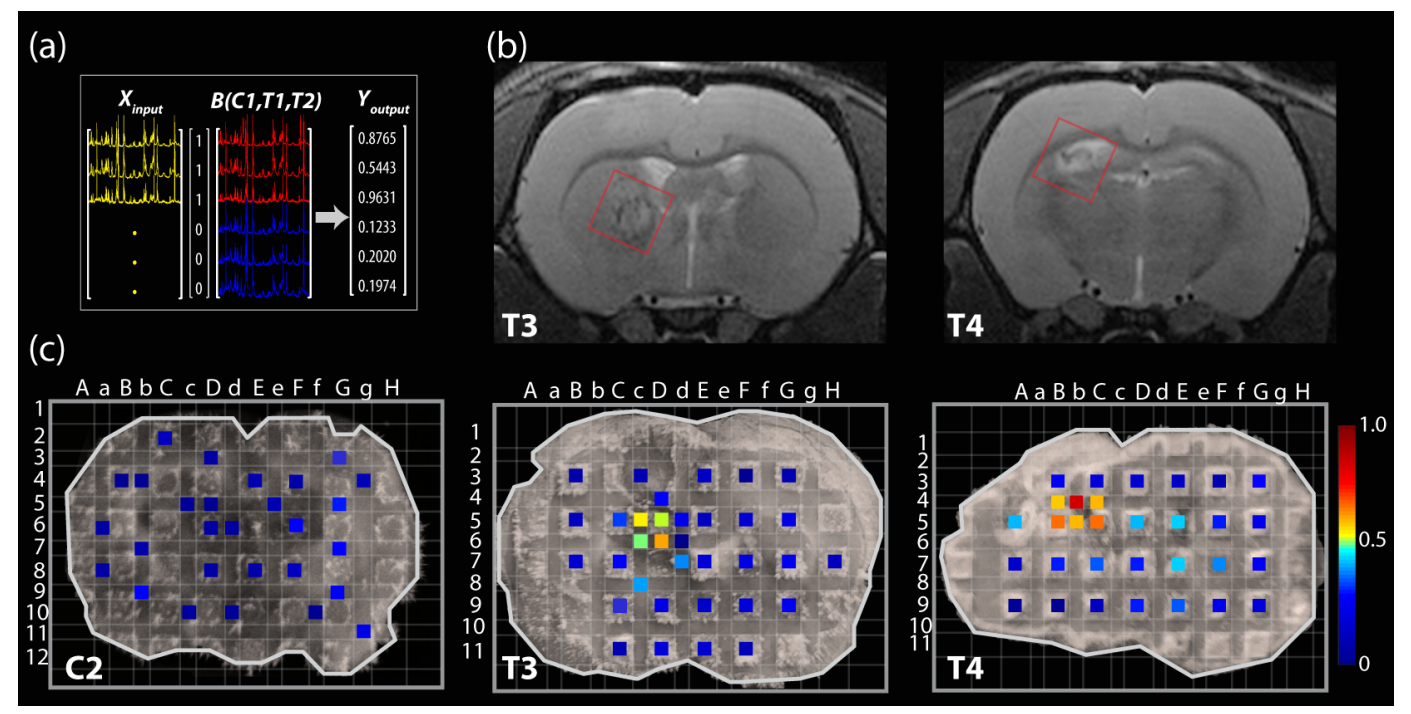

Figure 3

(a)

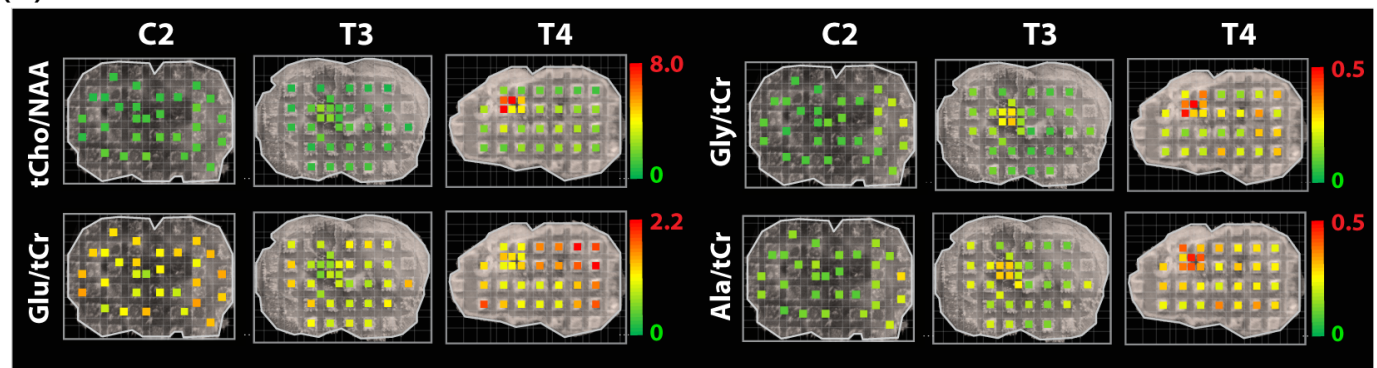

(b) ipsilateral $\quad \vdots$ contralateral ipsilateral $\quad \vdots$ contralateral ipsilateral $\quad \vdots \quad$ contralateral

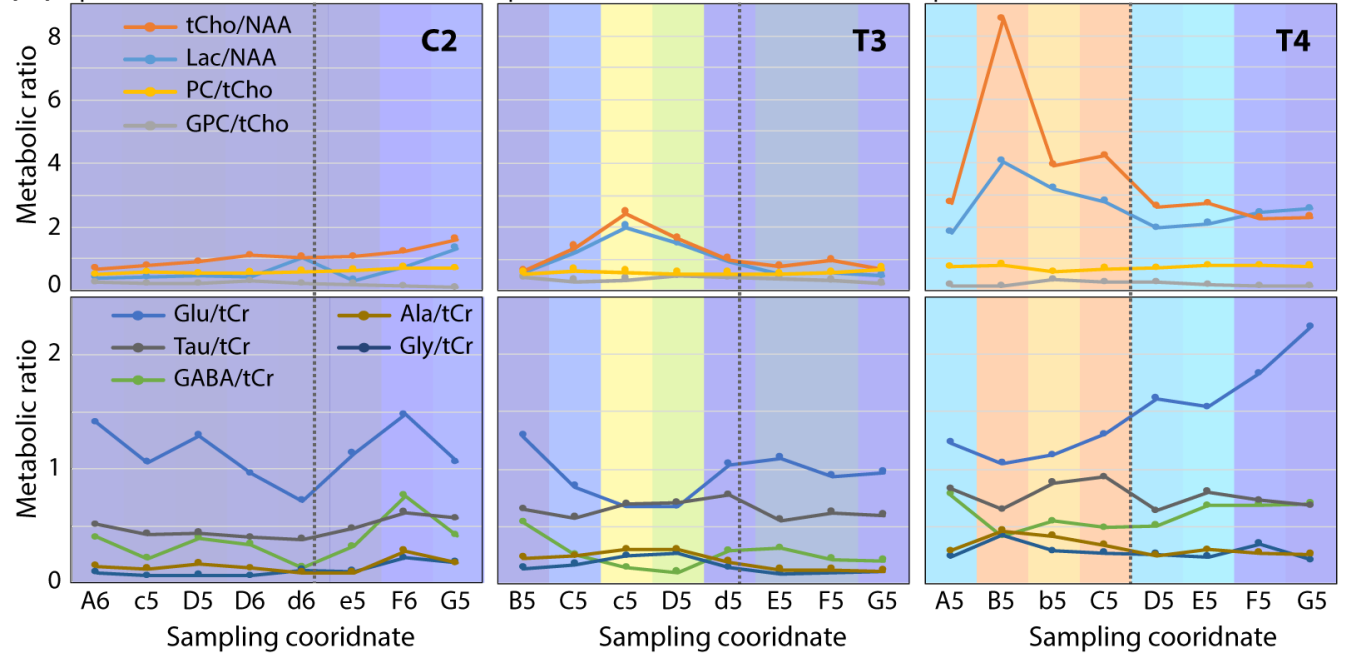

Figure 4 


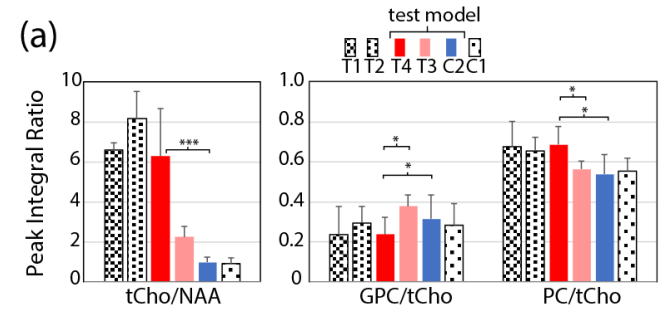

(b)
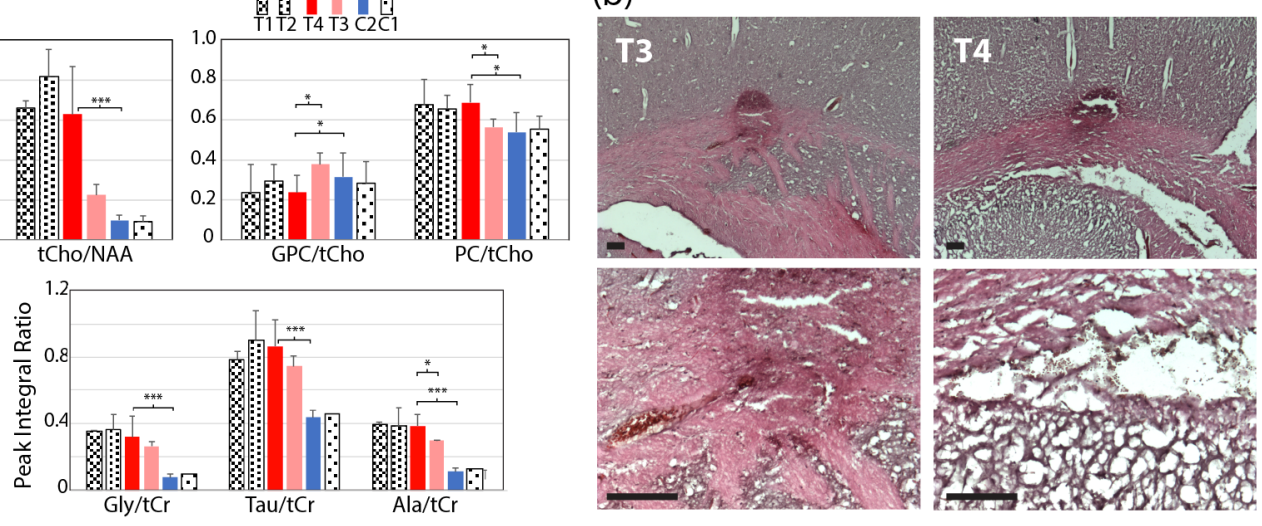

Figure 5 